\title{
Environment-dependent tight-binding potential model
}

\author{
M. S. Tang and C. Z. Wang* \\ Ames Laboratory-U.S. Department of Energy and Department of Physics, Iowa State University, Ames, Iowa 50011 \\ C. T. Chan \\ Ames Laboratory-U.S. Department of Energy and Department of Physics, Iowa State University, Ames, Iowa 50011 \\ and Physics Department, Hong Kong University of Science and Technology, Clear Water Bay, Hong Kong \\ K. M. Ho \\ Ames Laboratory-U.S. Department of Energy and Department of Physics, Iowa State University, Ames, Iowa 50011
}

(Received 25 August 1995)

\begin{abstract}
We present a tight-binding model which goes beyond the traditional two-center approximation and allows the hopping parameters and the repulsive energy to be dependent on the binding environment. Using carbon as an example, we show that the approach improves remarkably the transferability of the tight-binding model. The properties of the higher-coordinated metallic structures are well described by the model in addition to those of the lower-coordinated covalent structures.
\end{abstract}

Tight-binding molecular dynamics (TBMD) has recently been emerging as a useful and powerful scheme for atomistic simulation study of structural, dynamical, and electronic properties of realistic materials. ${ }^{1}$ The advantages of TBMD are that it includes explicit quantum-mechanical calculations into molecular dynamics and it is much faster than $a b$ initio methods. The scheme becomes even more attractive and promising due to recent developments in order- $N$ algorithms for electronic calculation and the use of parallel computers. ${ }^{2-5}$ Nevertheless, generating accurate and transferable tight-binding models for molecular dynamics simulation of realistic materials is still a very challenging task. In the last several years, a lot of effort has been devoted to the development of transferable tight-binding potentials. ${ }^{6-14} \mathrm{Re}-$ sults from these studies indicated that the tight-binding approach is quite successful for strongly bonded covalent systems such as carbon and silicon. However, even for these systems, the accuracy of existing models for the highercoordinated metallic structures (e.g., simple-cubic, $\beta$-tin, bcc, and fcc structures) are still far from satisfactory. ${ }^{1}$

We note that previous work on tight-binding potentials almost invariably adopts the two-center approximation for the hopping integrals. ${ }^{15}$ While the two-center approximation greatly simplifies the TB parametrization, neglecting multicenter interactions is justified only when the electrons are well localized in strong covalent bonds. For systems where metallic effects are significant, the two-center approximation is inadequate. In order to generate a tight-binding model that has good transferability over a wide range of coordination numbers, one can include multicenter interactions by allowing the interatomic interactions to depend on the binding environment.

In this paper, we present an approach that goes beyond traditional two-center approximations and allows the tightbinding parameters and the repulsive potential to be dependent on the bonding environment. We tested this model for the case of carbon and show that, in contrast to previous two-center models, the new approach describes properly the higher-coordinated metallic structures in addition to the diamond, graphite, and linear chain structures.

In this approach, the environment dependence of the hopping parameters is modeled through incorporating two new scaling functions into the traditional two-center integrals. The first one is a screening function, which mimics the electronic screening effects in solids such that the interaction strength between two atoms in the solid becomes weaker if there are intervening atoms located between them. This approach allows us to distinguish between first- and fartherneighbor interactions within the same interaction potential without having to specify separate interactions for first and second neighbors. The second function scales the distance between two atoms according to their effective coordination numbers. Longer effective bond lengths are assumed for higher-coordinated atoms. The strength of the hopping pa-

TABLE I. The parameters obtained from the fitting. The tight-binding parameters are in the unit of $\mathrm{eV}$ and the interatomic distances are in the unit of $\AA$. $\phi$ is dimensionless.

\begin{tabular}{lcccccccc}
\hline \hline & $\alpha_{1}$ & $\alpha_{2}$ & $\alpha_{3}$ & $\alpha_{4}$ & $\beta_{1}$ & $\beta_{2}$ & $\beta_{3}$ & $\delta$ \\
\hline$V_{s s \sigma}$ & -8.9491 & 0.8910 & 0.1580 & 2.7008 & 2.0200 & 0.2274 & 4.7940 & 0.0310 \\
$V_{s p \sigma}$ & 8.3183 & 0.6170 & 0.1654 & 2.4692 & 1.3000 & 0.2274 & 4.7940 & 0.0310 \\
$V_{p p \sigma}$ & 11.7955 & 0.7620 & 0.1624 & 2.3509 & 1.0400 & 0.2274 & 4.7940 & 0.0310 \\
$V_{p p \pi}$ & -5.4860 & 1.2785 & 0.1383 & 3.4490 & 0.2000 & 8.5000 & 4.3800 & 0.0310 \\
$\phi$ & 30.0000 & 3.4905 & 0.00423 & 6.1270 & 1.5035 & 0.205325 & 4.1625 & 0.002168 \\
$\Delta e_{s}, \Delta e_{p}$ & 0.79881 & 0.029681 & 0.19667 & 2.2423 & 0.055034 & 0.10143 & 3.09355 & 0.272375 \\
\hline \hline
\end{tabular}


TABLE II. The coefficients (in unit of eV) of the polynomial function $f(x)$.

\begin{tabular}{ccccc}
\hline \hline$c_{0}$ & $c_{1}$ & $c_{2}$ & $c_{3}$ & $c_{4}$ \\
\hline 12.201499972 & 0.583770664 & $0.336418901 \times 10^{-3}$ & $-0.5334093735 \times 10^{-4}$ & $0.7650717197 \times 10^{-6}$ \\
\hline \hline
\end{tabular}

rameters between atoms $i$ and $j$ is therefore dependent on the coordination number of the atoms: weaker interaction strength for larger-coordinated structures. This model preserves the two-center form of the tight-binding hopping integral while taking multicenter effects into account.

We use a minimal basis set of one $s$ and three $p$ atomic orbitals to construct the tight-binding Hamiltonian for carbon with the hopping parameters and the pairwise repulsive potential expressed as

$$
h\left(r_{i j}\right)=\alpha_{1} R_{i j}^{-\alpha_{2}} \exp \left[-\alpha_{3} R_{i j}^{\alpha_{4}}\right]\left(1-S_{i j}\right)
$$

In this expression, $h\left(r_{i j}\right)$ denotes the possible types of interatomic hopping integrals, $V_{s s \sigma}, V_{s p \sigma}, V_{p p \sigma}, V_{p p \pi}$, or pairwise repulsive potential $\phi\left(r_{i j}\right)$ between atoms $i$ and $j$. $r_{i j}$ is the real distance and $R_{i j}$ is the scaled distance between atoms $i$ and $j$ [see Eq. (4)]. $S_{i j}$ is a screening function. The parameters $\alpha_{1}, \alpha_{2}, \alpha_{3}$, and $\alpha_{4}$ and parameters for the scaling function $R_{i j}$ and the screening function $S_{i j}$ can be different for different hopping integrals and pairwise repulsive potential. These parameters will be determined in the fitting procedure. In general, we require that $S_{i j}$ is near 0 if $i$ and $j$ are nearest-neighbor atoms, and close to 1 otherwise so that nearest-neighbor interactions dominate. Note that expression (1) reduces to the traditional two-center form if we set $R_{i j}=$ $r_{i j}$ and $S_{i j}=0$.

The screening function is modeled as

$$
S_{i j}=\frac{\exp \left(\xi_{i j}\right)-\exp \left(-\xi_{i j}\right)}{\exp \left(\xi_{i j}\right)+\exp \left(-\xi_{i j}\right)},
$$

with

$$
\xi_{i j}=\beta_{1} \sum_{l} \exp \left[-\beta_{2}\left(\frac{r_{i l}+r_{j l}}{r_{i j}}\right)^{\beta_{3}}\right],
$$

where $\beta_{1}, \beta_{2}$, and $\beta_{3}$ are screening parameters. Note that $\xi_{i j}$ depends not only on the distance between atoms $i$ and $j$, but also on the positions of the neighbors of atoms $i$ and $j$. The maximum screening effect occurs when the atom $l$ is just sitting on the line connecting the atoms $i$ and $j$ $\left(r_{i l}+r_{l j}\right.$ is minimum). The screening function decays rapidly when the neighboring atoms move away from the line joining atoms $i$ and $j$. The screening function varies smoothly from 0 to near 1 as $\xi$ is increased.

The scaling between the real and effective interatomic distance is defined by
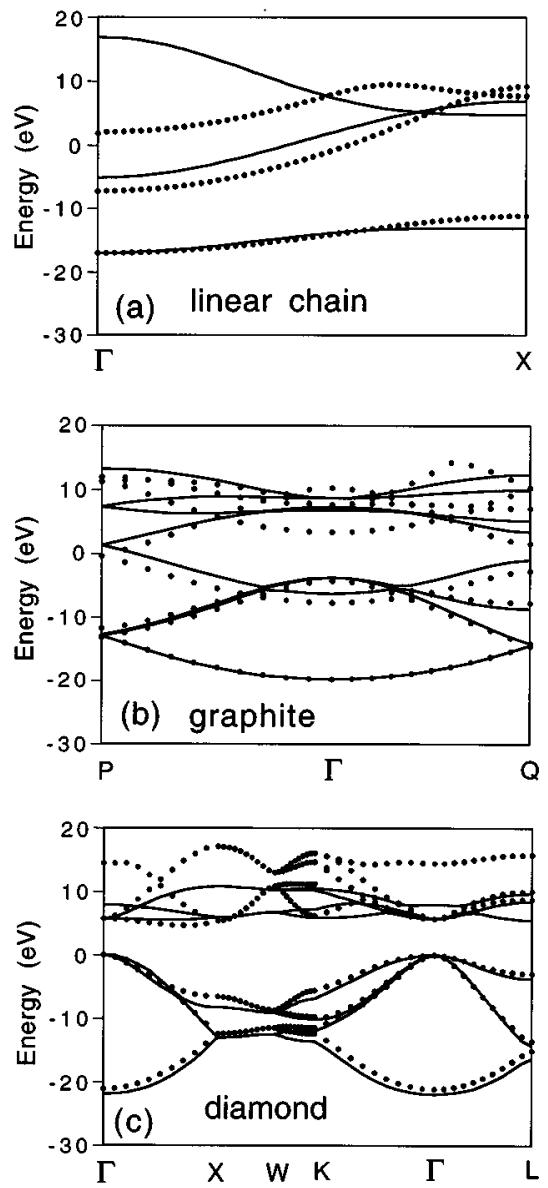
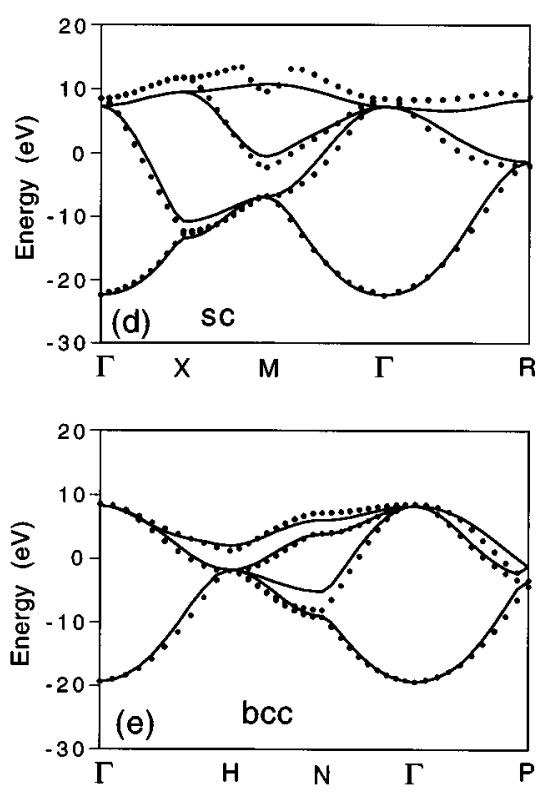

FIG. 1. The electronic energy bands of various crystalline structures of carbon calculated using the present TB model (solid curves) are compared with the first-principles LDA calculation results (dots). The Fermi levels are located around $E=0 \mathrm{eV}$.

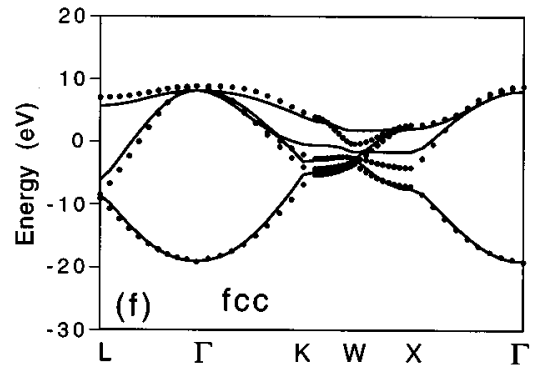



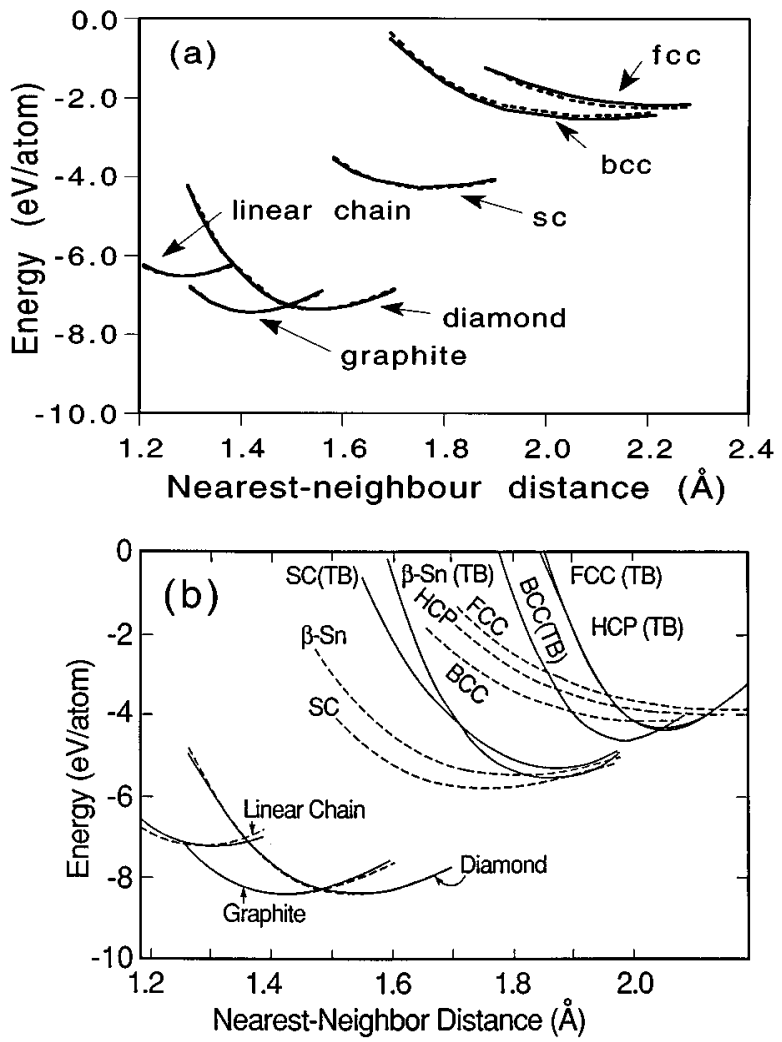

FIG. 2. (a) The binding energies as a function of nearest-neighbor distance for carbon in different crystalline structures calculated using the present TB model are compared with the results from the first-principles LDA (with gradient correction) calculations. The solid curves are the TB results and the dashed curves are the LDA results. (b) The results obtained from the two-center TB model of Ref. 9. The LDA results (dashed curves) in (b) are calculated without gradient correction.

$$
R_{i j}=r_{i j}\left\{1+\frac{\delta}{2}\left[\left(\frac{g_{i}-g_{0}}{g_{0}}\right)+\left(\frac{g_{j}-g_{0}}{g_{0}}\right)\right]\right\},
$$

where $g_{i}$ and $g_{j}$ are the effective coordination numbers of atoms $i$ and $j$, and $g_{0}$ denotes the coordination number for a reference atom in a reference structure. We will use the diamond structure as the reference structure for group IV elements.

TABLE III. Elastic constants, phonon frequencies, and Grünneisen parameters of diamond calculated from the present TB model are compared with previous TB model (Ref. 9) and experimental results (Ref. 18). Elastic constants are in units of $10^{12} \mathrm{dyn} / \mathrm{cm}^{2}$ and the phonon frequencies are in terahertz.

\begin{tabular}{lccc}
\hline \hline & $\begin{array}{c}\text { Present } \\
\text { model }\end{array}$ & $\begin{array}{c}\text { Previous } \\
\text { model }\end{array}$ & Experiment \\
\hline$a(\AA)$ & 3.585 & 3.555 & 3.567 \\
$B$ & 4.19 & 4.56 & 4.42 \\
$c_{11}-c_{12}$ & 9.25 & 6.22 & 9.51 \\
$c_{44}$ & 5.55 & 4.75 & 5.76 \\
$\nu_{\mathrm{LTO}(\mathrm{\Gamma})}$ & 41.61 & 37.80 & 39.90 \\
$\nu_{\mathrm{TA}(\mathrm{X})}$ & 25.73 & 22.42 & 24.20 \\
$\nu_{\mathrm{TO}(\mathrm{X})}$ & 32.60 & 33.75 & 32.0 \\
$\nu_{\mathrm{LA}(\mathrm{X})}$ & 36.16 & 34.75 & 35.5 \\
$\gamma_{\mathrm{LTO}(\mathrm{I})}$ & 0.93 & 1.03 & 0.96 \\
$\gamma_{\mathrm{TA}(\mathrm{X})}$ & 0.30 & -0.16 & \\
$\gamma_{\mathrm{TO}(\mathrm{X})}$ & 1.50 & 1.10 & \\
$\gamma_{\mathrm{LA}(\mathrm{X})}$ & 0.98 & 0.62 & \\
\hline \hline
\end{tabular}

TABLE IV. Elastic constants, phonon frequencies, and Grüneisen parameters of graphite calculated from the present TB model are compared with the previous TB model (Ref. 9) and experimental results (Ref. 19). Elastic constants are in units of $10^{12} \mathrm{dyn} / \mathrm{cm}^{2}$ and the phonon frequencies are in terahertz.

\begin{tabular}{lccc}
\hline \hline & $\begin{array}{c}\text { Present } \\
\text { model }\end{array}$ & $\begin{array}{c}\text { Previous } \\
\text { model }\end{array}$ & Experiment \\
\hline$c_{11}-c_{12}$ & 8.94 & 8.40 & 8.80 \\
$E_{2 g_{2}}$ & 48.99 & 49.92 & 47.46 \\
$A_{2 u}$ & 26.07 & 29.19 & 26.04 \\
$\gamma\left(E_{2 g_{2}}\right)$ & 1.73 & 2.00 & 1.63 \\
$\gamma\left(A_{2 u}\right)$ & 0.05 & 0.10 & \\
\hline \hline
\end{tabular}

The effective coordination number is given by

$$
g_{i}=\sum_{j}\left(1-S_{i j}\right)
$$

where $S_{i j}$ is also in the form of the screening function defined above (but with different parameters). Note that when $i$ and $j$ are nearest-neighbor atoms, $S_{i j}$ is close to 0 and $g_{i}$ counts almost one neighbor. On the other hand, $S_{i j}$ is close to 1 if $i$ and $j$ are not nearest-neighbor atoms, so that $g_{i}$ counts only a small fraction of a neighbor. $g_{i}$ defined as (5) thus provides a continuous and smooth function for counting the neighbors. In this paper, the parameters for $S_{i j}$ in Eq. (5) are chosen before the band structures and binding energies are fitted. These parameters are $\beta_{1}=2.0, \beta_{2}=0.0478$, and $\beta_{3}=7.16$. Using these parameters, $g_{i}$ are calculated to be $2.08639, \quad 3.17678,4.41022,6.23620,10.38529$, and 11.89829 for the linear-chain, graphite, diamond, simplecubic, bcc, and fcc structures, respectively. These values give a reasonable representation of the effective coordinations in these structures.

Besides the hopping parameters, the diagonal matrix elements in this model are also dependent on the bonding environments. The expression for the diagonal matrix elements is

$$
e_{\lambda, i}=e_{\lambda, 0}+\sum_{j} \Delta e_{\lambda}\left(r_{i j}\right)
$$

where $\Delta e_{\lambda}\left(r_{i j}\right)$ takes the same expression as Eq. (1) and $\lambda$ denotes the two types of orbitals ( $s$ or $p$ ). $e_{s, 0}$ and $e_{p, 0}$ are chosen to be -6.041 and $1.024 \mathrm{eV}$, respectively.

Finally, we express the repulsive energy term in a functional form as in the previous tight-binding model for carbon developed by $\mathrm{Xu}$ et al., ${ }^{9}$ that is,

$$
E_{\mathrm{rep}}=\sum_{i} f\left(\sum_{j} \phi\left(r_{i j}\right)\right),
$$

where $\phi\left(r_{i j}\right)$ is a pairwise potential between atoms $i$ and $j$ and $f$ is a functional expressed as a fourth-order polynomial with argument $x=\Sigma_{j} \phi\left(r_{i j}\right)$, i.e., $f(x)=\sum_{n=0}^{n=4} c_{n} x^{n}$.

The parameters in the model are determined by first fitting to the electronic band structures and then the cohesive energy versus volume curves for linear chain, graphite, diamond, simple cubic, bcc, and fcc structures, respectively obtained from the self-consistent first-principles densityfunctional calculations. The local density functional calculations were performed using the Ceperly-Alder local exchange-correlation energy as parametrized by Perdew and 
Zunger, ${ }^{16}$ gradient corrections for the exchange-correlation energy were also included following the generalized gradient approximation proposed by Perdew and Wang. ${ }^{17}$ The electronic wave functions were expanded in a mixed basis set containing plane waves up to an energy cutoff of 25 Ry and 4 localized orbitals per carbon atom. The sampling grid for Brillouin zone integration of the total-energy and electronic charge calculations contains $60,90,50,165,165$, and $146 k$ points, respectively, for the diamond, graphite, linear chain, and the simple cubic, bcc, and fcc structures.

The fitting is performed with heavier weight on the lowerenergy diamond, graphite, and linear chain structures (about 10 times the weight for bcc and fcc structures). Because the use of a minimal basis set is inadequate for describing the higher-energy bands, we focus our fitting on the occupied energy bands and some states above but near the Fermi level. Additional checks have also been made to ensure that the model gives reasonable results for the elastic moduli and phonon frequencies in the diamond and graphite structures. The parameters obtained from such fitting are listed in Tables I and II.

In Fig. 1, the band structures obtained from the present model for carbon are compared with the first-principles calculation results. The tight-binding model reproduces very well the occupied bands for carbon crystalline structures with coordination numbers varying from 2 (linear chain) to 12 (fcc). The lower part of the conduction bands in simplecubic, bcc, and fcc structures and the band gap in the diamond structure are also fairly well described. Due to the absence of higher-energy orbitals in the basis set, our model does not have a good fit to the conduction bands of the three lower-coordinated covalent structures. However, a good description of the occupied energy bands should be adequate for studying the total energies and structural properties of condensed phases.

The binding energies as a function of nearest-neighbor distance for carbon in different crystalline structures are presented in Fig. 2(a). Results from the previous tight-binding model of Xu et al. ${ }^{9}$ using the two-center approximation is also shown in Fig. 2(b) for the purpose of comparison. It is clear that the present tight-binding model improves significantly the transferability of the model to describe the energies of the metallic structures. The model also describes accurately the binding energy of the hexagonal diamond structure, which is about $0.2 \mathrm{eV} /$ atom higher than that of cubic diamond.

We have also calculated some phonon frequencies and elastic constants for diamond and graphite structures. The results are listed in Tables III and IV in comparison with the results of previous TB models and experimental values. The results also show overall improvements over the previous model using the two-center approximation. In particular, the elastic constants of diamond obtained from the present model are in much better agreement with the experimental data. The vibration frequencies calculated at zero temperature are slightly higher than the experiment values. Since the experimental values are obtained at room temperature, we believe that the vibration frequencies from the present tightbinding model should be in even better agreement with experiments if one takes into account temperature effects.

In summary, we show in this paper an empirical scheme to incorporate environment dependence of interactions into the tight-binding model. We demonstrated that the inclusion of three-center interactions into the tight-binding model improves the transferability of the model to describe the higher coordinated metallic structures. This approach is very successful in describing the band structures, banding energies, and elastic and vibrational properties of carbon structures with coordination numbers ranging from 2 to 12 . We anticipate that it will improve the transferabilities of the tightbinding models for silicon, germanium, and transition-metal elements.

Ames Laboratory is operated for the U.S. Department of Energy by Iowa State University under Contract No. W-7405-Eng-82. This work was supported by the Director for Energy Research, Office of Basic Energy Sciences, and the High Performance Computing and Communications initiative, including a grant of computer time at the NERSC at Livermore.
*Author to whom correspondence should be addressed.

${ }^{1}$ For review, see C. Z. Wang, K. M. Ho, and C. T. Chan, Comp. Mat. Sci. 2, 93 (1994); C. Z. Wang and K. M. Ho, Adv. Chem. Phys. (to be published).

${ }^{2}$ X.-P. Li, R. W. Nunes, and D. Vanderbilt, Phys. Rev. B 47, 10891 (1993).

${ }^{3}$ M. S. Daw, Phys. Rev. B 47, 10895 (1993).

${ }^{4}$ F. Mauri, G. Galli, and R. Car, Phys. Rev. B 47, 9973 (1993); F. Mauri and G. Galli, Phys. Rev. Lett. 73, 3471 (1994).

${ }^{5}$ S. Y. Qiu, C. Z. Wang, K. M. Ho, and C. T. Chan, J. Phys. Condens. Matter 6, 9153 (1994).

${ }^{6}$ L. Goodwin, A. J. Skinner, and D. G. Pettifor, Europhys. Lett. 9, 701 (1989).

${ }^{7}$ S. Sawada, Vacuum 41, 612 (1990).

${ }^{8}$ M. Kohyama, J. Phys. Condens. Matter 3, 2193 (1991).

${ }^{9}$ C. H. Xu, C. Z. Wang, C. T. Chan, and K. M. Ho, J. Phys. Condens. Matter 4, 6047 (1992).

${ }^{10}$ I. Kwon, R. Biswas, C. Z. Wang, K. M. Ho, and C. M. Soukoulis, Phys. Rev. B 49, 7242 (1994).
${ }^{11}$ J. L. Mercer, Jr. and M. Y. Chou, Phys. Rev. B 47, 9366 (1993).

${ }^{12}$ J. L. Mercer, Jr. and M. Y. Chou, Phys. Rev. B 49, 8506 (1994).

${ }^{13}$ K. Stokbro, N. Chetty, K. W. Jacobsen, and J. K. Norskov, Phys. Rev. B 50, 10727 (1994).

${ }^{14}$ R. E. Cohen, M. J. Mehl, and D. A. Papaconstantopoulos, Phys. Rev. B 50, 14694 (1994).

${ }^{15}$ J. C. Slater and G. F. Koster, Phys. Rev. 94, 1498 (1954).

${ }^{16}$ P. Perdew and A. Zunger, Phys. Rev. B 23, 5048 (1981).

${ }^{17}$ P. Perdew and Y. Wang, Phys. Rev. B 33, 8800 (1986).

${ }^{18}$ Semiconductors: Physics of Group IV Elements and III-V Compounds, Landolt-Börnstein New Series, Group III, Vol. 17, Pt. a, edited by O. Madelung, M. Schulz, and H. Weiss (SpringerVerlag, Berlin, 1982); Semiconductors: Intrinsic Properties of Group IV Elements and III-V, II-VI and I-VII Compounds, Landolt-Börnstein New Series, Group III, Vol. 22, Pt. a, edited by O. Madelung and M. Schulz (Springer-Verlag, Berlin, 1987).

${ }^{19}$ M. S. Dresselhaus and G. Dresselhaus, in Light Scattering in Solids III, edited by M. Cardona and G. Guntherodt (Springer, Berlin, 1982), p. 8. 